\title{
Concerns about the off-licence use of amiodarone for Ebola
}

\author{
Ankur Gupta-Wright academic clinical fellow in infectious disease ${ }^{1}$, James Lavers intensive care \\ specialist registrar ${ }^{2}$, Sharon Irvine clinical fellow (infectious diseases) ${ }^{3}$ \\ 'Department of Clinical Research, London School of Hygiene and Tropical Medicine, London WC1E 7HT, UK; ${ }^{2}$ Ysbyty Gwynedd, Bangor, UK \\ ${ }^{3}$ Institute of Infection, Immunity and Inflammation, University of Glasgow, Glasgow, UK
}

We were disturbed by your article promoting the compassionate use of amiodarone for Ebola virus disease by the non-governmental organisation (NGO), Emergency, which suggested its use was endorsed by the Department for International Development and WHO. ${ }^{1}$

Healthcare professionals working within the wider medical community in Sierra Leone have expressed serious concern about the off-licence use of this potentially toxic drug in suspected and confirmed Ebola without the patient's consent. Amiodarone has well documented cardiac, hepatic, and pulmonary side effects. ${ }^{2}{ }^{3}$ Evidence of benefit in Ebola virus disease is speculative at best, and amiodarone is not considered a priority novel drug for Ebola by WHO, further questioning the ethics of its use in the current outbreak. ${ }^{4}$ This use of amiodarone, and other unconventional practices-such as the use of high doses of diuretic in Ebola associated acute kidney injury—at Emergency's Ebola treatment centre reaffirms the pressing need for greater accountability and clinical governance of NGOs acting independently in medical disaster situations.

Competing interests: All authors previously volunteered as doctors with Emergency at the Lakka Ebola Treatment Centre in Sierra Leone.

1 Turone F. Doctors trial amiodarone for Ebola in Sierra Leone. BMJ 2014;349:g7198. (27 November.)

2 Chen C-C, Wu C-C. Acute hepatotoxicity of intravenous amiodarone: case report and review of the literature. Am J Ther 2014 Sep 24. [Epub ahead of print].

Range FT, Hilker E, Breithardt G, Buerke B, Lebiedz P. Amiodarone-induced pulmonary toxicity — a fatal case report and literature review. Cardiovasc Drugs Ther 2013;27:247-54

4 WHO. Potential Ebola therapies and vaccines. 2014. www.who.int/csr/resources/ publications/ebola/potential-therapies-vaccines/en/.

Cite this as: BMJ 2015;350:h272

๑ BMJ Publishing Group Ltd 2015 